\title{
WAVE ENERGY DISSIPATION BY ANISOTROPIC VISCOSITY IN MAGNETIC X-POINTS
}

\author{
I. J. D. CRAIG \\ Department of Mathematics, University of Waikato, Hamilton 3216, New Zealand; i.craig@waikato.ac.nz \\ AND \\ YuRi E. LiTVINENKo \\ Institute for the Study of Earth, Oceans, and Space, University of New Hampshire, \\ Durham, NH 03824; yuri.litvinenko@unh.edu \\ Received 2006 December 19; accepted 2007 June 22
}

\begin{abstract}
The viscous dissipation of axial field disturbances in planar magnetic X-points is examined. It is emphasized that an accurate treatment requires a nonisotropic tensor viscosity whose components are governed by the local magnetic field. Numerical solutions are constructed, which compare the buildup of viscous forces using the tensor formulation against a simplified model based on conventional shear viscosity. The scaling of the global energy-loss rate with the viscosity coefficient is shown to follow $P_{\nu} \sim \nu^{1 / 3}$ for both the traditional shear viscosity and the Braginskii bulk viscosity. This suggests that viscous wave dissipation can occur quite rapidly, in a few tens of Alfvén times. The results imply that large-scale disturbances, generated by magnetic reconnection in the solar corona, should dissipate in a time on the order of a few minutes and significantly contribute to coronal heating.
\end{abstract}

Subject headings: MHD — plasmas — Sun: flares — Sun: magnetic fields

\section{INTRODUCTION}

The problem of heating the solar corona to multimillion-kelvin temperatures remains a major challenge in space physics (see, e.g., Klimchuk 2006 for a recent review). It is generally accepted that photospheric plasma motions increase the free energy of the coronal magnetic field and thus provide the energy source for the observed heating. Yet the dominant mechanism of the energy release remains unidentified. Two basic mechanisms under consideration are direct electric current heating and wave energy damping. In this paper, we consider the wave dissipation mechanism.

Although upward-propagating waves carry enough energy to heat the corona, they are heavily damped by steep density and temperature gradients in the chromosphere and transition region. It is not clear what the solution to this difficulty is. Some studies suggest that torsional Alfvén waves can be transmitted from the photosphere to the corona efficiently enough to heat the corona (Kudoh \& Shibata 1999). Alternatively, once the energy is accumulated in stressed coronal magnetic fields in response to slowly changing boundary conditions at the photosphere, impulsive magnetic reconnection can generate waves in the corona itself, eliminating the transmission problem (see, e.g., Sturrock 1999). In order to develop this idea, wave energy dissipation rates in the vicinity of magnetic reconnection sites need to be determined. This is the goal of the present paper.

Since the classical electric resistivity is very small in the coronal plasma, conventional resistive models for wave damping require strongly localized electric currents. The plasma viscosity in the corona is also small, yet typical viscous coefficients parallel to the magnetic field exceed the resistivity coefficient by many orders of magnitude even when turbulent effects are taken into consideration. This is why viscous dissipation is likely to be a more effective mechanism of wave damping in the corona. The important role of viscosity in a wide range of coronal processes has been emphasized theoretically (Hollweg 1985).

Viscous-plasma dynamics and energetics are often modeled using the standard symmetric viscous stress tensor, which corre- sponds to the usual shear viscous force (e.g., Heyvaerts \& Priest 1983). Viscous stresses, however, are highly anisotropic in a magnetized plasma (Braginskii 1963; Hollweg 1986), and therefore significant viscous damping will depend strongly on the magnetic geometry. Previous studies of the Braginskii viscosity investigated in detail the dissipation associated with resonant absorption (e.g., Davila 1987; Hollweg \& Yang 1988; Ofman et al. 1994; Erdélyi \& Goossens 1995) and dissipation of waves propagating in a homogeneous plasma with a uniform background magnetic field (e.g., Porter et al. 1994). Here we focus on the role of anisotropic viscosity in the damping of shear waves in line-tied magnetic X-points. Specifically, we formulate an initial-value problem in which a planar magnetic X-point is perturbed by finite-amplitude disturbances normal to the plane. The present analysis extends the X-point study of Fruit \& Craig (2006), who employed only the conventional shear viscosity. Our results may provide a local description for interacting magnetic loops with footpoints anchored at the photosphere.

In $\S 2$, we give the mathematical formulation of the problem. We discuss the incompressible MHD equations and introduce the $2 \frac{1}{2}$-dimensional geometry that forms the backdrop of our analysis. Numerical results are presented in $\S 3$, where we discuss the velocity and magnetic field evolution and the damping of waves by viscous forces. We also compare scalings for anisotropic viscous damping against results based on conventional shear viscosity. Specifically, side-by-side computations are introduced to compare scalings for anisotropic viscous damping with results based on conventional shear viscosity. Possible effects of plasma compressibility on our results are explored in the Appendix. Conclusions are summarized in $\S 4$.

\section{VISCOUS DAMPING IN X-POINT GEOMETRIES}

\subsection{The Role of Viscous Effects}

To emphasize the importance of viscous effects in the solar atmosphere, consider a typical solar active region, characterized by a magnetic field $B_{0}=10^{2} \mathrm{G}$, plasma density $\rho_{0}=10^{-15} \mathrm{~g} \mathrm{~cm}^{-3}$, 
and size $L=10^{9.5} \mathrm{~cm}$. The corresponding Alfvén speed is $v_{\mathrm{A}}=$ $10^{9} \mathrm{~cm} \mathrm{~s}^{-1}$. Dimensionless measures of dissipation coefficients are obtained by scaling the resistivity and viscosity coefficients by $4 \pi v_{\mathrm{A}} L / c^{2}$ and $v_{\mathrm{A}} L$, respectively. The dimensionless resistivity is then an inverse Lundquist number of order $\eta \simeq 10^{-14.5}$, based on the classical value of resistivity $\sim T^{-3 / 2}$ for a coronal plasma temperature $T=10^{6} \mathrm{~K}$ (Spitzer 1962). For the same parameters in a fully ionized plasma, the dimensionless viscosity parallel to the magnetic field $\sim T^{5 / 2}$ is of order $\nu \simeq 10^{-4.5}$. Hence $\nu / \eta \simeq$ $10^{10} \gg 1$, strongly suggesting that viscous effects can be essential in the coronal dynamics and energetics (Hollweg 1985).

An important point is that viscous transport is highly anisotropic in the magnetized coronal plasma (Braginskii 1963; Hollweg 1986). Specifically, the viscous stress tensor is strongly modified when the proton mean free path exceeds the gyroradius: $\omega_{p} \tau_{p} \gg 1$, where $\omega_{p}=e B /\left(m_{p} c\right)$ is the proton cyclotron frequency and $\tau_{p} \simeq 0.75 T^{3 / 2} / n$ is the mean time (in seconds) between momentumchanging collisions. The above parameters lead to $\omega_{p} \tau_{p} \simeq 10^{6} \gg 1$, which confirms that an anisotropic viscosity should be employed in studies of coronal wave damping.

It is worth stressing from the outset that the Braginskii stress tensor contains a term proportional to the divergence of the plasma velocity. In the model we consider, which focuses on the damping of incompressible shear waves, this term is set to zero. Although there is no inconsistency in this treatment, we recognize that compressibility generally plays an important role in the wave dynamics by coupling various components of the wave velocity (Davila 1987). Moreover, strictly incompressible disturbances are not likely to be excited in a strongly structured solar corona (Davila 1987). It follows that our treatment of wave dynamics and dissipation may be justified only locally, say, in the vicinity of a magnetic null, where we can ignore the complicated geometry of multiple magnetic loops, which appear on a larger scale in the corona. It is also worth remembering that dissipation in the problem of resonant absorption is primarily determined by shear viscosity rather than by the dominant terms in the stress tensor (Hollweg \& Yang 1988; Ofman et al. 1994). Again, our neglect of the shear component of the Braginskii stress tensor is justified as long as the bulk viscosity effects are significant, which is indeed the case for the assumed form of the magnetic field disturbances.

\subsection{Incompressible MHD Equations}

In what follows, we investigate the damping of shear wave disturbances, normal to the plane of a magnetic X-point and line-tied at a rigid rectangular outer boundary. We adopt the $2 \frac{1}{2}$-dimensional MHD equations (e.g., Craig \& Litvinenko 2005). We use the stream function representation for the plasma velocity,

$$
\boldsymbol{v}(x, y, t)=\nabla \phi \times \hat{z}+W \hat{z},
$$

and the flux function representation for the magnetic field,

$$
\boldsymbol{B}(x, y, t)=\nabla \psi \times \hat{z}+Z \hat{z} .
$$

These forms identically satisfy the constraints that $\boldsymbol{v}$ and $\boldsymbol{B}$ be divergence-free.

It is convenient to work with the curled form of the momentum equation and to scale the system using typical magnitudes for the length, density, and magnetic field intensity. Velocities are then measured in units of the Alfvén speed and times are scaled by the Alfvén time. We employ Poisson brackets, typified by $[\psi, \phi]=\partial_{x} \psi \partial_{y} \phi-\partial_{y} \psi \partial_{x} \phi$, and write $\boldsymbol{F}$ for the viscous force. Ignoring gravity, the MHD equations reduce to the following system:

$$
\begin{gathered}
\partial_{t}\left(\nabla^{2} \phi\right)+\left[\nabla^{2} \phi, \phi\right]=\left[\nabla^{2} \psi, \psi\right]+G, \\
\partial_{t} \psi+[\psi, \phi]=\eta \nabla^{2} \psi, \\
\partial_{t} W+[W, \phi]=[Z, \psi]+F_{\perp}, \\
\partial_{t} Z+[Z, \phi]=[W, \psi]+\eta \nabla^{2} Z .
\end{gathered}
$$

Here

$$
G=-(\nabla \times \boldsymbol{F}) \cdot \hat{z}, \quad F_{\perp}=\boldsymbol{F} \cdot \hat{z}
$$

define planar and axial viscous contributions, while the electric current density $\eta \nabla^{2} \psi$ accounts for resistive decay. These equations are assumed to hold within the rectangular domain $(|x|,|y|) \leq 1$. Field lines are anchored on a boundary that is idealized as a highly conducting rigid surface on which the velocity field vanishes. This choice of boundary condition is synonymous with the assumption of line-tying at the photosphere. The photospheric plasma is so dense that coronal disturbances must vanish at the photospheric boundary. Line-tying is a standard choice for a boundary condition in studies of wave dynamics in the corona (e.g., Díaz et al. 2004).

Below we formulate the initial-value problem that describes the viscous damping of axial field disturbances $W(x, y, t)$ and $Z(x, y, t)$ in planar magnetic X-points.

\subsection{Viscous Stresses and Viscous Dissipation}

The MHD system described above has provided the basis for a wide class of analytical and numerical investigations, often involving the reconnection of field lines. In such cases the main focus is typically on the flux transfer afforded by the resistive term $\eta \nabla^{2} \psi$. On the other hand, viscosity is often either completely neglected without much justification or given a simplified classical description. In particular, if we write

$$
\boldsymbol{F}=\nabla \cdot \mathcal{S}
$$

for the viscous force, then the classical form for the shear viscosity $F_{i}=\partial_{j} \mathcal{S}_{i j}=\nu \nabla^{2} v_{i}$ is based on the symmetric tensor

$$
\mathcal{S}_{i j}=\nu\left(\partial_{j} v_{i}+\partial_{i} v_{j}-\frac{2}{3} \delta_{i j} \nabla \cdot \boldsymbol{v}\right) .
$$

As already mentioned, however, the viscosity becomes highly nonisotropic in the presence of a sufficiently strong magnetic field, necessitating a more accurate tensor description. If $\omega_{p} \tau_{p} \gg 1$, then the dominant terms in the viscous stress tensor are as follows:

$$
\mathcal{S}_{i j}=\nu_{0}\left(3 \frac{B_{i} B_{j}}{B^{2}}-\delta_{i j}\right)\left(\frac{B_{m} B_{k}}{B^{2}} \partial_{k} v_{m}-\frac{\nabla \cdot \boldsymbol{v}}{3}\right)
$$

with $\nu_{0} \simeq \nu$ (Braginskii 1963; Hollweg 1986). Equations (9) and (10) simplify somewhat in the incompressible limit $\nabla \cdot \boldsymbol{v}=0$, considered in this paper. Note, however, that the term involving $\nabla \cdot v$ is retained in the compressible viscous computations described in the Appendix.

The global viscous dissipation rate is given by

$$
P_{\nu}=\int \mathcal{S} \cdot \nabla \boldsymbol{v} d V
$$


In sharp contrast to the usual shear viscosity, the dominant terms in the viscous stress tensor in a magnetized plasma describe the bulk viscosity. In this case, viscous forces and dissipation rates depend sensitively on the magnetic field. One can see from equation (10) that a necessary condition for bulk viscous dissipation is that gradients of $\boldsymbol{v}$ along the field lines are nonvanishing, that is, $(\boldsymbol{B} \cdot \nabla) v \neq 0$. As a simple example, note that if the magnetic field is unidirectional, say, $\boldsymbol{B}=B \hat{\boldsymbol{z}}$, the dissipation rate reduces to $P_{\nu}=3 \nu_{0} \int\left(\partial_{z} W\right)^{2} d V$ (where $\left.W=\boldsymbol{v} \cdot \hat{z}\right)$. Clearly, viscous dissipation is absent unless the plasma is accelerated along the magnetic field lines. Although this may happen when flux-pileup magnetic reconnection is driven by vortical plasma flows (Litvinenko 2005), more generally we expect significant viscous dissipation only if strong corrugations of the magnetic field develop in the course of the evolution of the system.

\subsection{Axial Field Dissipation in Magnetic X-Points}

As a basis for all the subsequent discussion, we assume that finite-amplitude axial displacements are superposed on a static magnetic X-point:

$$
\psi=x y, \quad \phi=0
$$

We are interested in global initial disturbances, since these are the most difficult to dissipate efficiently but most likely to contain the bulk of the wave energy. The initial displacements satisfying the line-tying condition are taken to be

$$
Z_{0}=0, \quad W_{0}=\sin \pi x \sin \pi y .
$$

To explore the effect of the magnetic field geometry, we also consider wave damping near a one-dimensional neutral sheet,

$$
\psi=\frac{1}{2} y^{2}, \quad \phi=0,
$$

with the corresponding initial conditions

$$
Z_{0}=0, \quad W_{0}=\cos \frac{1}{2} \pi x \sin \pi y .
$$

Previous studies of viscous wave damping in these geometries assumed that the shear viscosity provides the dominant damping mechanism (Craig \& Fruit 2005; Fruit \& Craig 2006). It has been shown that for long-wavelength axial disturbances, there is an initial quasi-ideal phase in which sharp cross-field gradients on length scales on the order of $\delta l \sim \nu^{1 / 3}$ are built up by phase mixing (Heyvaerts \& Priest 1983). This is followed by a stage of strong wave damping, in which energy is rapidly dissipated, $P_{\nu} \sim \nu W^{2} /(\delta l)^{2} \sim \nu^{1 / 3}$. The later asymptotic decay can be quite complicated, and it is possible for self-similar modes to develop in which there is a breakdown of energy equipartition. But since the bulk of the wave energy is lost within 20 or 30 Alfvén times (for $\nu \simeq 10^{-4.5}$ ), before the asymptotic phase sets in, the global energetics is largely unaffected.

We now ask, how effective is the Braginskii (anisotropic) viscosity in damping the global wave energy? We assume velocity and magnetic fields of the form

$$
\boldsymbol{B}=\left(B_{x}, B_{y}, Z(x, y, t)\right), \quad \boldsymbol{v}=(0,0, W(x, y, t)),
$$

with $B_{x}=x$ and $B_{y}=-y$ for the X-point geometry (eq. [12]). We calculate the axial viscous force using

$$
F_{\perp}=\partial_{x} \mathcal{S}_{31}+\partial_{y} \mathcal{S}_{32}
$$

and for the case of the X-point field the forms in equation (16) lead to

$$
\begin{aligned}
F_{\perp}=3 \nu_{0}\left\{\partial_{x}\right. & {\left[x \frac{Z^{2}}{B^{4}}\left(x \partial_{x} W-y \partial_{y} W\right)\right] } \\
+ & \left.\partial_{y}\left[y \frac{Z^{2}}{B^{4}}\left(y \partial_{y} W-x \partial_{x} W\right)\right]\right\} .
\end{aligned}
$$

The corresponding expression in the case of the neutral sheet (eq. [14]) is as follows:

$$
F_{\perp}=3 \nu_{0} y^{2} \partial_{x}\left(\frac{Z^{2}}{B^{4}} \partial_{x} W\right) .
$$

These expressions for $F_{\perp}$ are used to obtain numerical solutions for $W(x, y, t)$ and $Z(x, y, t)$ assuming that anisotropic viscous damping is the dominant dissipation mechanism, $\nu_{0} \gg \eta$. Note that in the case of the one-dimensional sheet, we expect that the buildup of sharp cross-field corrugations by phase mixing should have little effect on the resulting viscous force, since gradients in $y$ are not represented in equation (19). A further point is that close to a null point (or line), the magnetic field vanishes and the Braginskii viscous tensor is not valid. Hence it is legitimate to augment $F_{\perp}$ with a term describing the classical shear viscosity in the vicinity of the null. In fact, the computations presented below are generally insensitive to the shear components acting close to the null, at least as far as X-point wave energy damping is concerned (see $\S 3.2$ ).

Figure 1 shows a typical example of the corrugated field structure that develops as a result of the quasi-ideal X-point evolution for the case of anisotropic viscosity with $\nu_{0}=10^{-3}$ and $\eta=10^{-5}$. The surface plots for the axial wave fields $Z$ and $W$ were taken around the time of maximum energy dissipation rate. For $\nu_{0} \gg \eta$, this time $(t \simeq 9)$ approximately coincides with the time of maximum viscous forces on the plasma. Figure 2, which shows the corresponding contour plots, confirms that the developing corrugations are aligned with planar projections of the X-point field lines. Quite similar corrugated structures are observed when the shear viscosity is substituted for the Braginskii form (see Fruit \& Craig 2006).

\section{SIMULATION OF THE AXIAL FIELD EVOLUTION}

\subsection{Viscous Dissipation in the X-Point Field}

In the results that follow, we demonstrate how the buildup of viscous forces in the plasma leads to significant rates of energy dissipation. Since the corrugated wave field profiles of Figures 1 and 2 derive from the effects of quasi-ideal phase-mixing processes, similar structures are achieved for both tensor and shear viscosities. The time taken to achieve the peak dissipation rate does, however, depend on the strength and form of the viscous damping.

To obtain quantitative results, we compare the time evolution of the global wave energy,

$$
\mathcal{E}(t)=\int \frac{1}{2}\left(Z^{2}+W^{2}\right) d V,
$$

using side-by-side computations for the bulk and shear viscosity. We compute the $W$ - and $Z$-fields from

$$
\partial_{t} W=[Z, \psi]+F_{\perp}, \quad \partial_{t} Z=[W, \psi]+\eta \nabla^{2} Z,
$$



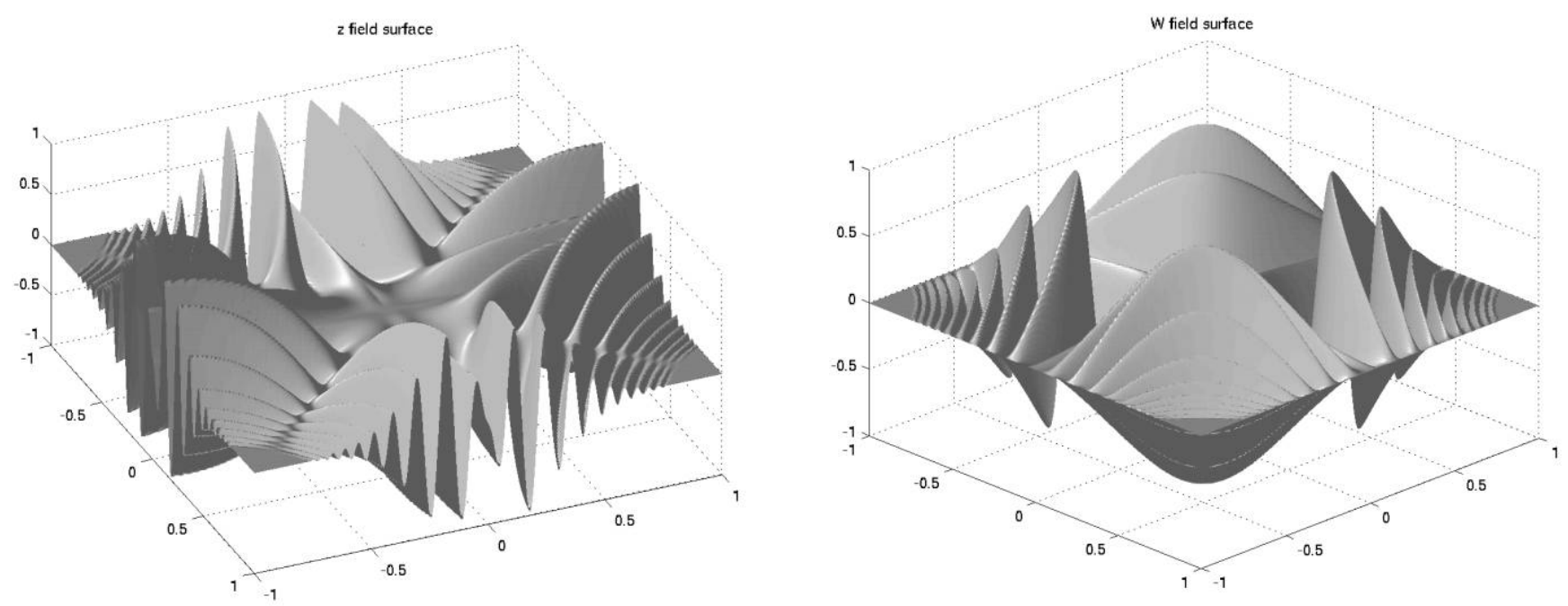

Fig. 1.- Surface plots of the axial magnetic field $Z$ and the axial velocity field $W$ taken at the time of maximum viscous dissipation rate $(t=9)$, using the Braginskii viscosity for $\nu_{0}=10^{-3}$ and $\eta=10^{-5}$.

which follow from equations (5) and (6) on assuming the forms in equation (16). The viscous force $F_{\perp}$ is derived from the X-point and current-sheet fields, as discussed in $\S 2.4$.

Figure 3 provides energy-loss rates for the $\mathrm{X}$-point for the parameters $\nu_{0}=\nu=10^{-3} \gg \eta$. The global dissipation rates are physically significant in both cases, but the viscous forces build more slowly and there is a modest reduction in the peak damping rate when the nonisotropic viscosity is introduced. This behavior is common to all simulations with $\nu_{0}=\nu \gg \eta$.

Figure 4 summarizes dissipation scalings based on peak energyloss rates for the range $10^{-4} \leq \nu=\nu_{0} \leq 10^{-2}$ with $\eta=10^{-2} \nu$. Note that the lower limit corresponds to the viscosity in a coronal plasma with temperature around $10^{6} \mathrm{~K}$. Perhaps the most important observation is that the Braginskii viscosity retains the scaling $P_{\nu} \sim \nu^{1 / 3}$ for the global energy-loss rate associated with the shear viscosity.

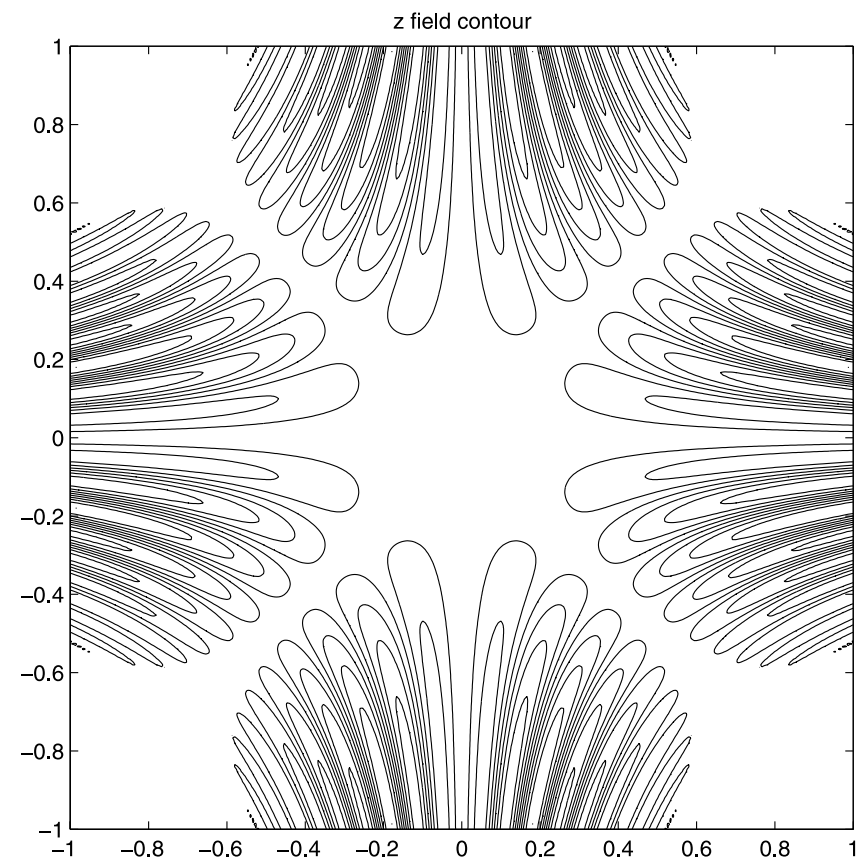

Given that the conventional shear viscosity is not represented in the dominant terms of the Braginskii tensor, it is perhaps surprising that the anisotropic bulk viscosity is so effective at dissipating the X-point wave energy. According to the scalings of Figure 4, peak rates are reduced by only a factor of 3 , compared with those for the unrealistic shear viscosity. Yet the physics of energy dissipation is quite different: evidently the strong corrugations in the magnetic field, formed during the quasi-ideal phase of the evolution, provide multiple locations where the plasma can flow along the local magnetic field - and these are just the conditions required for rapid wave damping by bulk viscosity (see discussion at the end of $\S 2.3$ ).

It is important to remember that all the present results are based on the field decomposition of equation (16). This form is justified for the shear viscosity because planar and axial field disturbances decouple as long as the disturbances remain incompressible. The

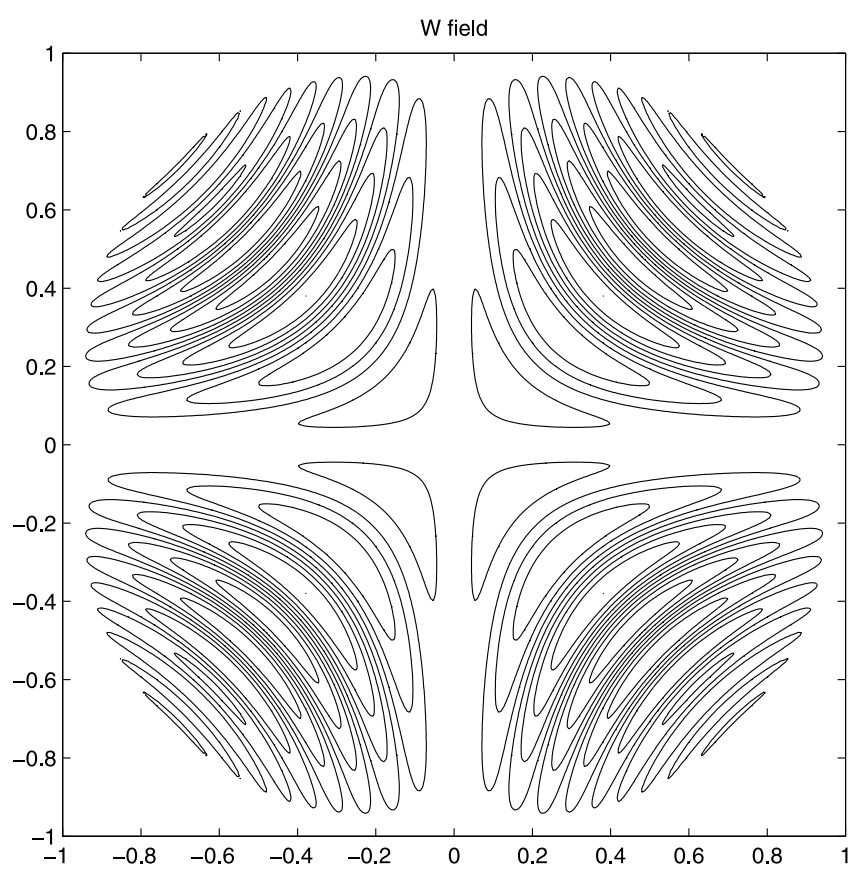

FIG. 2.-Contour plots of the axial magnetic field $Z$ and the axial velocity field $W$ for the parameters of Fig. 1 . 


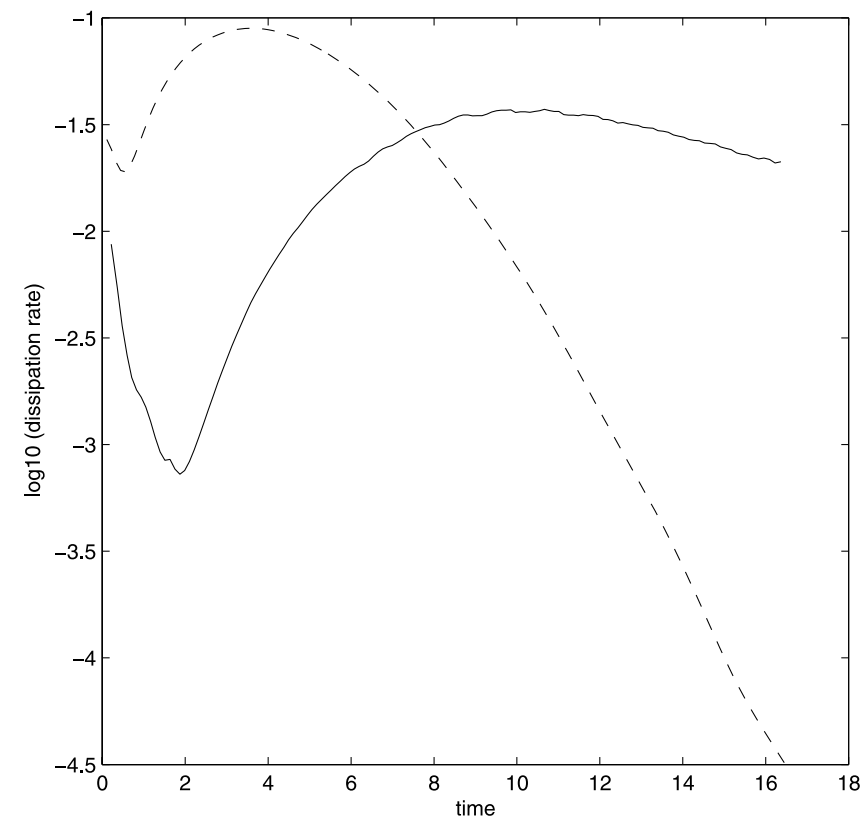

FIG. 3.-Viscous dissipation rates in the Braginskii (solid line) and shear (dashed line) viscosity computations for the two-dimensional X-point $\left(\nu_{0}=\nu=10^{-3}, \eta=0\right)$.

Braginskii viscous stresses, on the other hand, induce planar viscous forces even when only axial flows are present initially. In this case, we assume that the induced planar fields can be regarded as secondary effects that derive from the buildup of viscous forces during the initial phases. This simplification, which corresponds to neglecting the viscous contribution $G$ in equation (3), is valid as long as axial flows with dimensionless speeds of order unity (that derive from the initial conditions) remain large compared with induced planar flows with speeds of order $\nu_{0} \ll 1$. This argument is supported by the close agreement of the incompressible and compressible X-point runs, described in the Appendix. The extent to which the neglect of secondary planar flows is justified in later stages of the wave evolution will be investigated elsewhere.

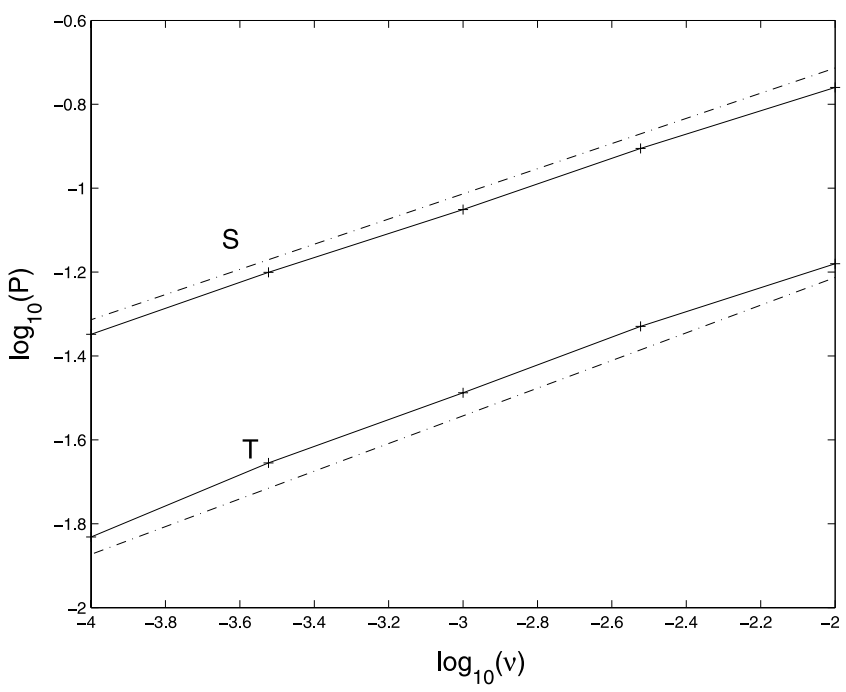

Fig. 4.-Scaling of the peak wave energy dissipation rate with viscosity ("S" indicates shear, " $\mathrm{T}$ " indicates tensor viscosity). The dot-dashed lines correspond to the power laws $P_{\nu} \simeq 0.77 \nu^{0.30}$ (shear viscosity, upper line) and $P_{\nu}=0.28 \nu^{0.33}$ (Braginskii viscosity, lower line). In all cases we have taken $\eta=10^{-2} \nu$.

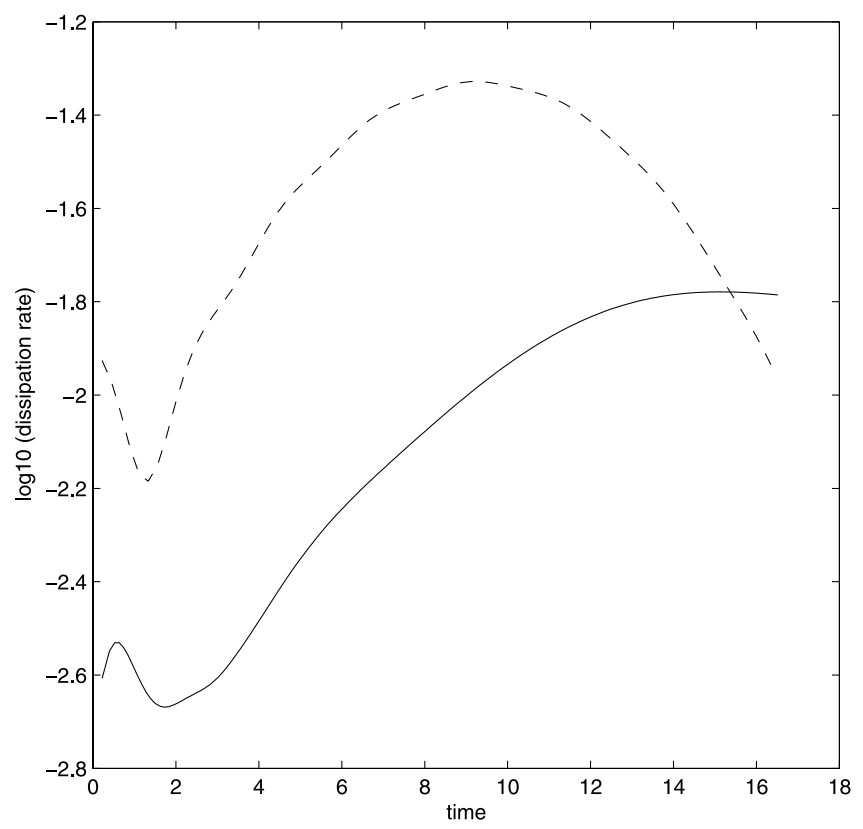

FIG. 5.- Same as Fig. 3, but for the one-dimensional current sheet.

\subsection{Influence of the Field Geometry}

We now turn to the influence of the field geometry on the viscous dissipation mechanism. As already mentioned, the currentsheet geometry (eq. [14]) is expected to reduce wave damping by the Braginskii viscosity quite significantly. Figure 5 is based on the same parameters as Figure 3 and confirms that the buildup of the viscous force and the peak dissipation rate are both weak when compared with the shear viscosity simulation. Even so, contrary to the argument following equation (19), there is a clear buildup in the dissipation rate as sharp gradients develop by phase mixing.

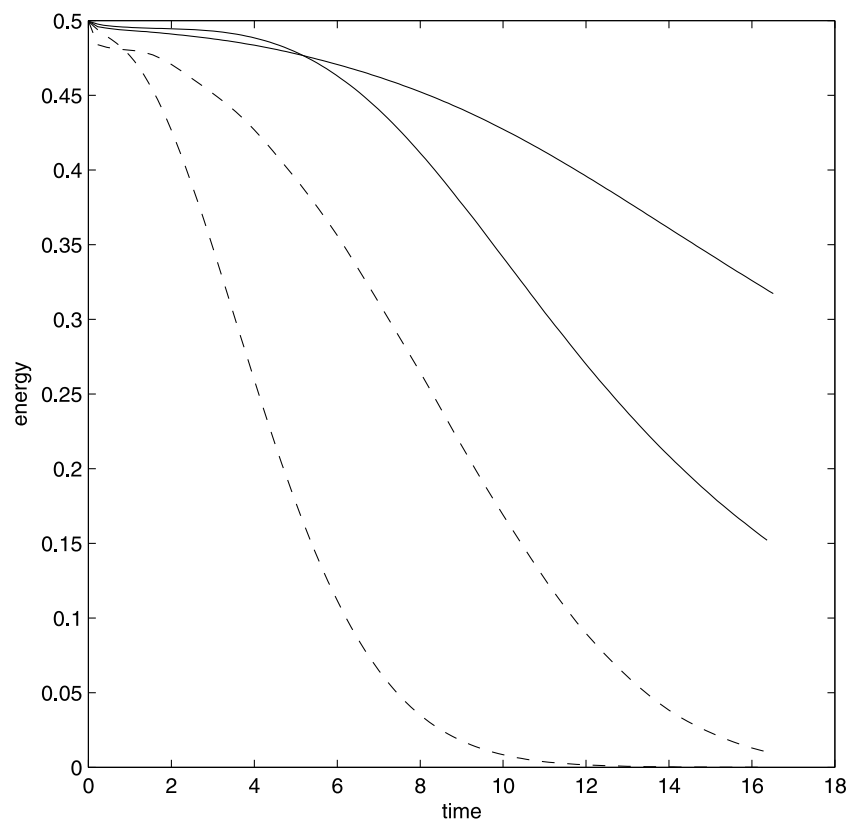

FIG. 6.- Global energy decay, based on the viscous wave-damping computations of Figs. 3 and $5\left(\nu=10^{-3}, \eta=0\right)$. Solid and dashed lines show results for the bulk and shear viscosity, respectively. In each case, the X-point geometry provides a more effective wave-damping model. The bulk viscosity model in the current-sheet geometry provides the weakest damping. 
This effect can be traced back to the residual influence of shear viscosity acting in the narrow weak-field region overlying the neutral line $y=0$. When only the bulk viscosity contribution in the computation is represented, this buildup is eliminated. Incidentally, shear viscosity components are less of an issue in the X-point computation because any residual shear viscous contribution is restricted only to a small circular region of negligible measure overlying the neutral point.

Figure 6 summarizes the global energy evolution in both X-point and current-sheet geometries. Shear and bulk viscosities are similarly effective in removing the bulk of the wave energy in around 20 Alfvén times for the X-point plasma. For the one-dimensional current sheet, however, damping by the bulk viscosity appears to be relatively ineffective. This conclusion is strengthened when it is remembered that shear viscosity components acting in the vicinity of the neutral line account for increases in the currentsheet dissipation rate.

\section{DISCUSSION}

We have considered the viscous damping of shear Alfvén waves in magnetic X-point geometries. The principal new result of this paper is the demonstration that shear waves in the vicinity of a magnetic reconnection site can be damped within 20 or 30 Alfvén times when realistic bulk viscosity is the primary dissipation mechanism. This rate corresponds to an energy decay time of a few minutes for conditions typical of the solar corona. Therefore, bulk viscous dissipation of waves, generated in the corona by magnetic reconnection or other mechanisms, can significantly contribute to coronal heating. The only caveat regarding this result is that the underlying magnetic geometry should not be overly simple. For instance, our examples show that wave damping in a two-dimensional X-point geometry is considerably more effective than in the one-dimensional current-sheet field.

In connection with the rate of the wave energy release, we note that anomalously enhanced dissipation coefficients are sometimes invoked in order to interpret the observed energy release in the corona. It appears unlikely though that turbulent resistivity or viscosity could change our estimates based on the classical values. It can be argued that the turbulent resistivity at coronal reconnection sites is enhanced by no more than a factor of $10^{6}$ relative to the classical value (e.g., Litvinenko \& Craig 2000 and references therein), which is still many orders of magnitude less than the classical viscosity coefficient. As far as turbulent viscosity is concerned, it can be estimated as $(\delta B / B)^{2} v_{\mathrm{A}}^{2} \tau_{c}$, where $\delta B$ is a characteristic amplitude of turbulent fluctuations and $\tau_{c} \simeq \omega_{p}^{-1}$ is a coherence time (Tsuda 1967). Given the smallness of the parameter $\left(\omega_{p} \tau_{p}\right)^{-1} \simeq 10^{-6}$ in the corona (see $\left.\S 2.1\right)$, it is clear that even a high turbulence level will not lead to enhancement of the viscous coefficient with respect to the classical value employed in this paper. What should be kept in mind, however, is that although bulk viscosity plays the dominant role in dissipation in our problem, this conclusion could change if we considered a more general problem of evolution of waves with nonvanishing compressible components. For instance, in studies of viscous dissipation by resonance absorption it is the shear viscosity, comparable in magnitude to the classical electric resistivity, that determines the rate of energy dissipation (e.g., Hollweg \& Yang 1988; Ofman et al. 1994). Even so, at least for the X-point damping problem, the results summarized in the Appendix suggest that the present computations are relatively robust to the inclusion of compressible effects.

Somewhat surprisingly, the key scaling $P_{\nu} \sim \nu^{1 / 3}$ for the maximum rate of viscous energy dissipation turns out to be independent of whether the usual shear viscosity or the Braginskii stress tensor is adopted. We conclude that in the latter case, rapidly formed magnetic field corrugations play the central role in creating large viscous stresses and ensuring comparable dissipation scalings. What should be investigated in the future is the validity of the assumption that the induced planar flows do not significantly influence the damping of the initially imposed axial flows - the decoupling assumption made in $\S 3.1$. We will address this question elsewhere.

This work was supported by the NSF Space Weather Program (grant ATM 05-19249), by the NASA Solar and Heliospheric Physics Supporting Research and Technology program (grant NNX07AI04G), by the NASA Living with a Star program (grant NNG05GM43G), and by the Marsden Fund (02-UOW-050 MIS). The authors would like to thank Professor Roger Hosking for discussions on the form of the viscous stress tensor.

\section{APPENDIX}

\section{COMPRESSIBLE VERSUS INCOMPRESSIBLE EVOLUTION}

In this paper, we analyzed the damping of shear waves in the incompressible approximation, setting from the outset the divergence of the plasma velocity to zero. Because compressibility can generally play an important role in the wave dynamics in the solar corona (e.g., Davila 1987), we have also performed a series of compressible runs, based on the polytropic law $p=k \rho^{\gamma}$ with plasma $\beta$ 's in the range $0 \leq \beta \leq 1$ and $\gamma \simeq 1$. In general, it is difficult to make a definitive comparison with the incompressible computations because compressible models involve additional phenomena that depend on both the wave perturbation amplitudes and the details of the pressure profiles. Qualitatively, we find compressible structures that mimic the highly corrugated profiles of the incompressible computations and global dissipation rates that are comparable to the rates associated with the incompressible bulk viscosity.

There is an important limiting case, however, that does allow a quantitative, side-by-side comparison with the bulk viscosity computation, namely, the evolution of low-amplitude axial disturbances in a highly compressible plasma of negligible gas pressure. Since the equations for the axial fields are formally identical in both models, it is possible to use an identical set of run parameters (those of $\$ 2.4$ ). Of course, the compressible computation incorporates the $\nabla \cdot \boldsymbol{v}$ term in the viscous stress tensor (eq. [10]), as well as the "secondary" planar field that is generated by the planar components of the viscous force. Hence, the coupling of the axial and planar fields is explicitly modeled in the compressible computation.

Figure 7 shows results based on a typical side-by-side comparison. It is clear that, at least for the initial phases of interest, the energy dissipation profiles for each model are virtually indistinguishable. The dashed and solid lines show the evolution of the global magnetic and kinetic energies for the compressible and incompressible runs, respectively. The profiles match so closely that, to facilitate a visual comparison, we have adjusted the initial conditions to allow for a slight separation of the decay curves. Similar computations confirm that the dissipation scalings, associated with the incompressible bulk viscosity computation (Fig. 4), are closely matched by the compressible model. 


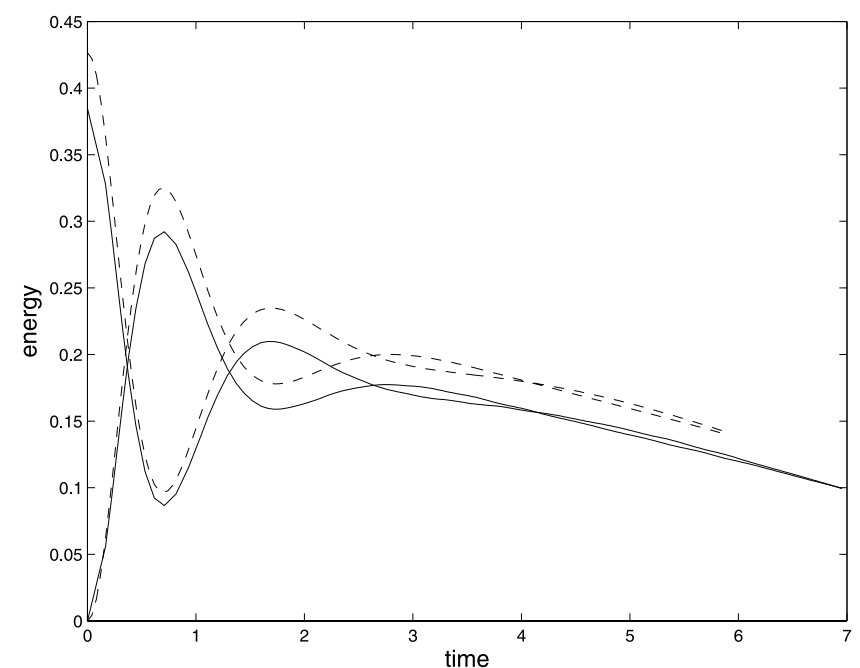

FIG. 7.- Typical time profiles of the global magnetic and kinetic energies for the incompressible (solid lines) and compressible (dashed lines) viscous tensor models $\left(\nu=5 \times 10^{-3}, \eta=10^{-4}\right)$. The gas pressure is negligible in the compressible computation. Although the energy initially resides in the axial velocity field, a significant fraction is transferred to the magnetic field over 1 Alfvén time. Once energy equipartition has set in, there is a steady monotonic decline in both components.

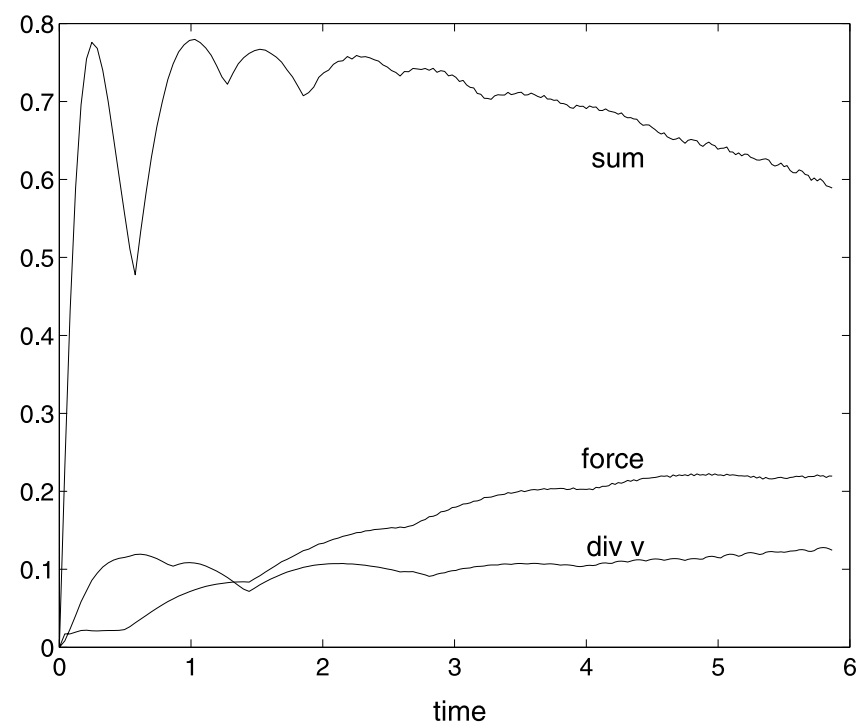

FIG. 8. - Gradual buildup of viscous forces in the compressible computation. Also shown are relative individual contributions to the viscous stress tensor. The relative compressible contribution $\nabla \cdot \boldsymbol{v}$ remains only a small fraction of the incompressible contribution $\left(B_{m} B_{k} / B^{2}\right) \partial_{k} v_{m}$ throughout the evolution. 
Figure 8 shows the buildup of the viscous forces in the compressible computation. Also shown are time traces of individual contributions to the compressible viscous stress tensor (eq. [10]), namely, the maximum $\nabla \cdot v$ over the mesh and the maximum of the sum $\left(B_{m} B_{k} / B^{2}\right) \partial_{k} v_{m}$. The fact that $\nabla \cdot v$ makes only a minor contribution to the tensor confirms that compressibility does not have a major impact on the energy decay. The same applies to the transfer of axial wave energy into the planar wave components, an effect neglected in the incompressible bulk viscosity computation (see $\S 3.1$ ).

These results suggest that the assumption of incompressiblity does not compromise the viscous dissipation model in any significant manner, at least in the case of low-amplitude axial perturbations in planar magnetic geometries in a uniform background plasma.

\section{REFERENCES}

Braginskii, S. I. 1963, Vopr. Teor. Plasmy, 1, 193 (English transl. Rev. Plasma Phys., 1, 205 [1965])

Craig, I. J. D., \& Fruit, G. 2005, A\&A, 440, 357

Craig, I. J. D., \& Litvinenko, Yu. E. 2005, Phys. Plasmas, 12, No. 112105

Davila, J. M. 1987, ApJ, 317, 514

Díaz, A. J., Oliver, R., Ballester, J. L., \& Roberts, B. 2004, A\&A, 424, 1055

Erdélyi, R., \& Goossens, M. 1995, A\&A, 294, 575

Fruit, G., \& Craig, I. J. D. 2006, A\&A, 448, 753

Heyvaerts, J., \& Priest, E. R. 1983, A\&A, 117, 220

Hollweg, J. V. 1985, J. Geophys. Res., 90, 7620 1986, ApJ, 306, 730
Hollweg, J. V., \& Yang, G. 1988, J. Geophys. Res., 93, 5423

Klimchuk, J. A. 2006, Sol. Phys., 234, 41

Kudoh, T., \& Shibata, K. 1999, ApJ, 514, 493

Litvinenko, Yu. E. 2005, Sol. Phys., 229, 203

Litvinenko, Yu. E., \& Craig, I. J. D. 2000, ApJ, 544, 1101

Ofman, L., Davila, J. M., \& Steinolfson, R. S. 1994, ApJ, 421, 360

Porter, L. J., Klimchuk, J. A., \& Sturrock, P. A. 1994, ApJ, 435, 482

Spitzer, L., Jr. 1962, Physics of Fully Ionized Gases (2nd rev. ed.; New York: Wiley)

Sturrock, P. A. 1999, ApJ, 521, 451

Tsuda, T. 1967, J. Geophys. Res., 72, 6013 